\title{
Thermality of the Hawking flux
}

\author{
Matt Visser \\ School of Mathematics, Statistics, and Operations Research, \\ Victoria University of Wellington, PO Box 600, Wellington 6140, New Zealand \\ E-mail: matt.visser@msor.vuw.ac.nz
}

AbStRaCT: Is the Hawking flux "thermal"? Unfortunately, the answer to this seemingly innocent question depends on a number of often unstated, but quite crucial, technical assumptions built into modern (mis-)interpretations of the word "thermal". The original 1850's notions of thermality — based on classical thermodynamic reasoning applied to idealized "black bodies" or "lamp black surfaces" - when supplemented by specific basic quantum ideas from the early 1900's, immediately led to the notion of the black-body spectrum, (the Planck-shaped spectrum), but without any specific assumptions or conclusions regarding correlations between the quanta. Many (not all) modern authors (often implicitly and unintentionally) add an extra, quite unnecessary, assumption that there are no correlations in the black-body radiation; but such usage is profoundly ahistorical and dangerously misleading. Specifically, the Hawking flux from an evaporating black hole, (just like the radiation flux from a leaky furnace or a burning lump of coal), is only approximately Planck-shaped over an explicitly bounded range of frequencies. Standard physics (phase space and adiabaticity effects) explicitly bound the frequency range over which the Hawking flux is approximately Planck-shaped from both above and below - the Hawking flux is certainly not exactly Planckian, and there is no compelling physics reason to assume the Hawking photons are uncorrelated.

KEYwords: Black Holes, Models of Quantum Gravity, Spacetime Singularities

ARXIV EPRINT: 1409.7754 


\section{Contents}

1 Introduction 1

2 Spectrum of the Hawking flux 2

3 Schwarzschild black holes 4

4 Connecting future and past null infinities 5

$\begin{array}{lll}5 & \text { Effective temperature } & 7\end{array}$

$\begin{array}{llr}6 & \text { Wick rotation and the Hawking flux } & 8\end{array}$

$\begin{array}{llr}7 & \text { Correlations in the Hawking flux } & 8\end{array}$

8 Analogue Hawking flux $\quad 9$

9 Discussion $\quad 10$

\section{Introduction}

Stephen Hawking predicted, (now some 40 years ago), that semi-classical black holes will emit quantum radiation with a temperature proportional to their surface gravity, and will slowly evaporate due to subtle quantum effects [1-3]. Direct experimental tests of this phenomenon have so far been impractical, and the best laboratory data comes from analogue systems such as surface waves in a water tank $[4,5]$, and more recently, phonons in a BEC [6]. Faced with this extreme paucity of both experimental and observational data, the community has focussed almost entirely on gedanken-experiments, with well over 5000 theory papers generated to date.

While there is almost universal agreement that the predicted Hawking flux will actually occur for a general relativistic black hole, and almost universal agreement that the associated back-reaction will slowly reduce the black hole's mass, there is relatively little agreement (in fact, considerable disagreement) regarding the endpoint of the Hawking evaporation process. In particular, the question of the thermality of the Hawking flux, (and the precise sense in which it is thermal), is a crucial and important ingredient in the so-called "information puzzle", and its more recent "firewall" variant [7-19]; this has important implications regarding the quantum unitarity of the Hawking evaporation process [20-24]. A key point is this: there is a crucial difference between the "qualitative" and "quantitative" information loss problems. 
- The "qualitative" problem is this: if a spacelike singularity forms (in the strict mathematical sense), then there will be a (strict mathematical) event horizon, and unavoidably some loss of unitarity associated with any matter that might cross the event horizon. ${ }^{1}$

- The "quantitative" problem is this: How much information is lost behind the event horizon, (if it forms), and how much comes out in the Hawking radiation?

Many authors have argued that no information comes out in the Hawking radiation, but that is rather begging the question. Other authors only address part of the puzzle. This subtlety has often been lost in the sometimes heated exchange of comments and criticisms. There is another way of saying this:

- Hawking radiation is associated with the apparent/trapping horizon, and couldn't care less about the event horizon (if present).

- Unitarity violation (if present) is associated with the event horizon (if present), and couldn’t care less about the apparent/trapping horizon.

Only if you assume that the event horizon actually forms, and that it closely tracks the apparent/trapping horizon, is there ever any significant information loss. To expand on these issues, let us first consider the spectral shape, and then the correlation structure, of the Hawking flux.

\section{Spectrum of the Hawking flux}

Hawking's 1974 derivation [1, 2], the many and quite varied subsequent re-derivations thereof, and the modern adiabatic variants of Hawking's original calculation [26, 27], all agree that the shape of the spectrum is approximately a Planckian black-body spectrum, but with certain key modifications and limitations. Specifically, the Planckian shape of the Hawking spectrum will, at an absolute minimum, be modified by at least three distinct physical effects:

1. greybody factors;

2. adiabaticity constraints;

3. available phase space.

Let us consider these three effects in turn.

\footnotetext{
${ }^{1}$ In his 1976 article [3] on the breakdown of predicability, Hawking phrased the discussion in terms of "hidden surfaces", reserving the phrase "event horizons" specifically for black holes. By doing things in this way his discussion applied also to the branching-off of baby universes, or indeed any sort of nontrivial temporal topology associated with what is now typically called a "causal horizon". For the purposes of this current article I will focus on the event horizons (possibly) associated with physical black holes, and the apparent/trapping horizons definitely associated with physical black holes [25]. Hawking's 1976 argument will apply whenever there is a causally inaccessible region in which one can hide correlations. Certainly that argument applies to the event horizons (possibly) associated with physical black holes.
} 
Greybody factors: these well-known effects arise when the Hawking flux is backscattered by the non-trivial gravitational field between the quasi-local horizon (the apparent/trapping horizon) and spatial infinity; the existence of these greybody effects is entirely standard and well-known, though quantitative estimates are sometimes tricky. Early work dates back to the mid-to-late 1970's [28-30], and interest in these quantities is active and ongoing, see for instance [31-33]. A key feature is to note that the greybody factors will always suppress the Hawking flux.

Adiabaticity constraints: these lesser-known effects arise from including back-reaction, and depend on the fact that the spacetime geometry must be slowly evolving (on the timescale set by the frequency of the Hawking photon) in order for Hawking's calculation, or any of its more modern variants, to apply. Implications of the adiabaticity condition were carefully analyzed and discussed in both references [26] and [27]. Specifically, to obtain an approximately Planckian spectrum the surface gravity must be "slowly evolving" in the sense that:

$$
|\dot{\kappa}| \ll \kappa^{2} .
$$

This is the constraint that a photon at the peak of the Hawking spectrum should not see any significant change in the surface gravity during one oscillation period.

More generally and quantitatively, let us now consider the conditions for the validity of Hawking's "exponential approximation" for the relative $e$-folding of the affine null parameters between past and future null infinity $\left(\mathrm{scri}^{-}\right.$and scri $\left.{ }^{+}\right)[1,2]$. Whenever the surface gravity is time-varying, $\dot{\kappa} \neq 0$, then the exponential approximation is at best valid over a bounded time interval of width $[26,27]$ :

$$
\Delta t \ll \frac{1}{\sqrt{|\dot{\kappa}|}} .
$$

See the discussion surrounding equation (10) of reference [26] or equation (3.22) of reference [27] for extensive technical details. But then Hawking's argument, (or its more modern variants), can be applied only to wave packets which can be localized within this time interval. This implies the wave-packet must be built out of modes of frequency at least $\omega_{\min }=\sqrt{|\dot{\kappa}|}$. In particular, a suitable extension of Hawking's argument leading to a Planckian spectrum only works for the limited range of frequencies

$$
\omega \gtrsim \omega_{\min }=\sqrt{|\dot{\kappa}|}
$$

Thus this adiabaticity argument provides an infrared frequency cutoff on the Hawking flux. If Hawking's argument (suitably extended) is to apply not just in the exponential Boltzmann tail, but also to include the peak of the Planck spectrum, then one recovers the quantitative condition $|\dot{\kappa}| \ll \kappa^{2}$.

Phase space effects: though typically ignored, there is also a phase space ultraviolet frequency cutoff in the Hawking flux. At its crudest, the emitted photon energy can never exceed the available mass energy: $\omega<m$. A slightly safer statement, for charged or rotating black holes, is $\omega<m-m_{\text {extremal }}$. 
More carefully, applying (standard flat-space) Lorentz kinematics in the far distant asymptotically flat region, it is easy to see that for a black hole of mass $m_{i}$ emitting, (in its rest frame), a photon of energy $\omega$, and thereby reducing its mass to $m_{f}$, one has:

$$
\omega=\frac{m_{i}^{2}-m_{f}^{2}}{2 m_{i}}
$$

Applied to the photons in the Hawking flux this yields the purely kinematic bound:

$$
\omega \leq \frac{m^{2}-m_{\text {extremal }}^{2}}{2 m}
$$

Thus this argument provides an ultraviolet frequency cutoff on the Hawking flux. ${ }^{2,3}$

Combined effects: combining these adiabaticity and phase space constraints, and noting the existence of grey-body effects, we can make the quantitative statement that the Hawking flux can (at best) be approximately Planckian only over the limited frequency range:

$$
\omega \in\left(\sqrt{|\dot{\kappa}|}, \frac{m^{2}-m_{\text {extremal }}^{2}}{2 m}\right) .
$$

Even within this range, where the (suitably extended modern variants of the) Hawking calculation can be trusted, greybody factors (barrier transmission probabilities) will to some extent suppress the Hawking flux below that of an ideal Planck spectrum.

\section{Schwarzschild black holes}

Let us now see what this quantitatively implies for Schwarzschild black holes: for the specific case of the Schwarzschild black hole $m_{\text {extremal }}=0$, so the phase space cutoff is simply $\omega<m / 2$. Indeed, for Schwarzschild black holes the phase space cutoff never intersects the peak $(\omega \sim \kappa)$ of the approximately Planck-shaped spectrum while one remains within the semi-classical regime.

\footnotetext{
${ }^{2}$ My own early views on the importance of the phase-space cutoff can be found in reference [34]. Although I am no longer in favour of the particular way that I discretized black hole entropy in that article, the comments regarding the importance of the phase-space cutoff and the final "particle cascade" leading to complete evaporation of Planck-scale black holes still hold.

${ }^{3}$ More recently the Parikh-Wilczek approach to Hawking radiation viewed as quantum tunnelling also explicitly (but somewhat indirectly) includes at least some phase-space effects and also adds nonlinear frequency-dependent terms in the action [35]. Parikh and Wilczek consider the emission of spherically symmetric thin shells, so 3-momentum conservation is trivial, and the phase space cutoff simplifies to $\omega \leq m-m_{\text {extremal }}$. For the Schwarzschild black hole Parikh and Wilczek find: $\operatorname{Im}($ Action $)=4 \pi \omega m\left(1-\frac{\omega}{2 m}\right)$, and relate this to $\Delta$ (Entropy) $=8 \pi \omega m\left(1-\frac{\omega}{2 m}\right)$, subject to $\omega \leq m$. Some authors prefer to interpret this as a frequency-dependent temperature, $T_{\text {effective }}(\omega)=T_{\text {Hawking }} \times\left(1-\frac{\omega}{2 m}\right)^{-1}$. The situation for ReissnerNordström black holes is considerably more subtle. There

$$
\Delta(\text { Entropy })=2 \pi\left\{\omega(2 m-\omega)-(m-\omega) \sqrt{(m-\omega)^{2}-q^{2}}+m \sqrt{m^{2}-q^{2}}\right\}, \quad \text { subject to } \omega \leq m-|q| .
$$

If desired, an effective temperature can be defined by $T_{\text {effective }}(\omega)=\omega / \Delta$ (Entropy), with a low-frequency expansion $T_{\text {effective }}(\omega)=T_{\text {Hawking }}+\mathcal{O}(\omega)$. Thus the Parikh-Wilczek approach provides both an explicit phase-space cutoff, and a modified emission amplitude.
} 
Because the phase space cutoff is so high, (compared to the location of the Hawking peak at $\omega \sim \kappa$ ), it is perfectly acceptable, (at least as a zeroth-order approximation), to approximate the Hawking flux by a complete Planck spectrum, integrate over all of phase space, and so get the Stefan-Boltzmann law, $\left(\dot{m}=-\sigma T^{4} A_{\text {horizon }}\right)$. But I emphasise that the applicability of the Stefan-Boltzmann law is intrinsically an approximate result; in view of the physical arguments presented above it cannot be exact.

Then, introducing Planck quantities for simplicity, we have $\kappa \sim m_{P}^{2} / m$. Thereby we deduce:

$$
\dot{\kappa}=-\frac{\dot{m} m_{P}^{2}}{m^{2}}=\left\{\left(\frac{m_{P}^{2}}{m}\right)^{4} \times 4 \pi(2 m)^{2}\right\} \frac{m_{P}^{2}}{m^{2}} \sim \frac{m_{P}^{6}}{m^{4}} .
$$

Consequently the Hawking flux from a Schwarzschild black hole is approximately Planckian (up to greybody factors) over the rather broad frequency interval

$$
\omega \in\left(\frac{m_{P}^{3}}{m^{2}}, \frac{m}{2}\right) .
$$

This interval is certainly non-empty for macroscopic black hole masses, and even for mesoscopic black hole masses all the way down to the Planck scale. Furthermore

$$
\frac{|\dot{\kappa}|}{\kappa^{2}} \sim \frac{m_{P}^{6} / m^{4}}{m_{P}^{4} / m^{2}}=\frac{m_{P}^{2}}{m^{2}} .
$$

So, as claimed, the peak of the Planck blackbody spectrum is indeed contained in the approximately Planckian interval all the way down to the Planck scale, (where one should stop believing semiclassical physics anyway).

Thus we see that for a Schwarzschild black hole these three bounds on the Planckian nature of the Hawking flux are (numerically) not particularly stringent. But they do however provide important qualitative information - at the very least they serve as a suitable antidote to the often made, (and often repeated, but utterly incorrect), assertion that the Hawking flux is exactly Planckian.

\section{Connecting future and past null infinities}

Let $U$ be an affine coordinate on past null infinity, while $u$ is taken to be an affine coordinate on future null infinity. Much of the physics of the Hawking effect is encoded in the $e$-folding relation connecting past and future null infinities $[1,2]$

$$
U=U_{H}-A \exp \left(-\kappa_{H} u\right) .
$$

Once one includes the effects of a time-dependent evolving black hole one should instead write $[26,27]$

$$
U(u)=U_{0}+\int_{u_{0}}^{u} \exp \left(-\int_{u_{0}}^{\bar{u}} \kappa(\tilde{u}) \mathrm{d} \tilde{u}\right) \mathrm{d} \bar{u} .
$$

Here $u_{0}$ is merely some convenient starting point, often taken to be the onset of black hole formation. If we approximate $\kappa(u) \approx \kappa_{0}$ as a constant then

$$
U(u) \approx U_{0}+\frac{1}{\kappa_{0}}-\frac{\exp \left(-\kappa_{0}\left[u-u_{0}\right]\right)}{\kappa_{0}},
$$

which is equivalent to the naive result used in the original 1974 calculations [1, 2]. 
Now let us make this more explicit and quantitative: when including the effects of back-reaction, for an evolving Schwarzschild black hole of initial mass $m_{0}$ we have $\kappa_{0} \sim$ $m_{P}^{2} / m_{0}$, and from the previous section $\dot{\kappa} \sim m_{P}^{-2} \kappa^{4}$. We can write this more carefully as the exact scaling relations $\kappa=\kappa_{0}\left(m_{0} / m\right)$ and $\dot{\kappa}=\dot{\kappa}_{0}\left(\kappa / \kappa_{0}\right)^{4}$, or even $\dot{\kappa}(u)=B \kappa(u)^{4}$, where approximately $B \sim m_{P}^{-2}$. Thereby

$$
\kappa(u)=\frac{\kappa_{0}}{\sqrt[3]{1-3 B \kappa_{0}^{3}\left[u-u_{0}\right]}} .
$$

This approximation will remain valid until the surface gravity rises to the Planck scale, which will happen when

$$
\frac{m_{P}^{3}}{m^{3}} \sim 1-3\left[u-u_{0}\right] \frac{m_{P}^{4}}{m^{3}} .
$$

That is when

$$
u-u_{0} \sim \frac{T_{P}}{3}\left(\frac{m^{3}}{m_{P}^{3}}-1\right) \sim \frac{T_{P}}{3} \frac{m^{3}}{m_{P}^{3}} .
$$

During that entire interval, from $u=u_{0}$ to $u \sim u_{0}+\frac{1}{3} T_{P}\left(m^{3} / m_{P}^{3}\right)$, the surface gravity (while not constant) is still slowly varying, in the sense of satisfying the adiabaticity constraint. In this interval $\kappa(u)$ can be integrated to explicitly yield

$$
\int_{u_{0}}^{u} \kappa(u) \mathrm{d} u=\frac{1}{2 B \kappa_{0}^{2}}\left[1-\sqrt[3]{1-3 B \kappa_{0}^{3}\left[u-u_{0}\right]}\right] .
$$

A second integration now gives

$$
\begin{aligned}
U(u)=U_{0} & +\frac{1}{\kappa_{0}}-\sqrt{2 B} D\left(\frac{1}{\sqrt{2 B} \kappa_{0}}\right) \\
& -\frac{1}{\kappa(u)} \exp \left(\frac{1}{2 B}\left[\frac{1}{\kappa(u)^{2}}-\frac{1}{\kappa_{0}^{2}}\right]\right) \\
& +\sqrt{2 B} \exp \left(\frac{1}{2 B}\left[\frac{1}{\kappa(u)^{2}}-\frac{1}{\kappa_{0}^{2}}\right]\right) D\left(\frac{1}{\sqrt{2 B} \kappa(u)}\right) .
\end{aligned}
$$

Here $D(x)$ is the Dawson function

$$
D(x)=e^{-x^{2}} \int_{0}^{x} e^{t^{2}} \mathrm{~d} t
$$

If desired the Dawson function can (up to rescaling) be related to the error function for imaginary argument, but in this real form is more suited to numeric manipulations. In particular the Dawson function is bounded by $D(x)<0.54105$, which means that the terms involving the Dawson function never shift $U(u)$ by more than one Planck time, and so can quietly be neglected until one reaches the Planck regime.

So for all practical purposes

$$
U(u)=U_{0}+\frac{1}{\kappa_{0}}-\frac{1}{\kappa(u)} \exp \left(\frac{1}{2 B}\left[\frac{1}{\kappa(u)^{2}}-\frac{1}{\kappa_{0}^{2}}\right]\right)+\mathcal{O}\left(T_{P}\right) .
$$

Note that while the relationship between future and past null infinity is now quite considerably more complicated than the simple $e$-folding of references $[1,2]$, we see that it is 
nevertheless quite explicit. Furthermore, at any particular time $u_{*}$ one can always locally approximate the exact $U(u)$ with an $e$-folding expression of the form $[26,27]$

$$
U\left(u \approx u_{*}\right) \approx U_{*}+\frac{1}{\kappa\left(u_{*}\right)}-\frac{\exp \left(-\kappa\left(u_{*}\right)\left[u-u_{*}\right]\right)}{\kappa\left(u_{*}\right)} .
$$

This formalism now gives one a slowly evolving Hawking temperature, at least until the mass of the black hole drops sufficiently low so that one enters the Planck regime.

\section{Effective temperature}

The net effect of these greybody factors, adiabaticity constraints, and phase space constraints is to modify the spectrum of the Hawking flux:

$$
n(k)=\frac{f(\omega)}{\exp \left(\hbar \omega / k_{B} T_{H}\right)-1} ; \quad f(\omega) \in(0,1) .
$$

Here $f(\omega)$ is some dimensionless suppression factor now encoding all three effects. This allows one (in the quite usual manner) to define an effective temperature in terms of the total energy flux:

$$
\sigma T_{\text {effective }}^{4}=\int n(k) \hbar \omega \frac{\mathrm{d}^{3} k}{(2 \pi)^{3}} .
$$

Setting $z=\hbar \omega / k_{B} T_{H}$, and $f(\omega) \rightarrow f(z)$, one sees

$$
T_{\text {effective }}^{4}=\frac{\int \frac{f(z) z^{3}}{e^{z}-1} \mathrm{~d} z}{\int \frac{z^{3}}{e^{z}-1} \mathrm{~d} z} T_{H}^{4} \leq T_{H}^{4} .
$$

That is, $T_{\text {effective }} \leq T_{H}$, so the effective temperature (bolometrically defined) of the outgoing Hawking flux has been suppressed below the naive Hawking temperature.

This then changes (both qualitatively and quantitatively) the entropy budget in the Hawking evaporation process. As the black hole evaporates, its Bekenstein entropy [36] decreases as

$$
\mathrm{d} S_{B}=-\frac{|\mathrm{d} M|}{T_{H}},
$$

whereas the entropy change of the outgoing radiation can best be estimated as

$$
\mathrm{d} S_{H}=+\frac{|\mathrm{d} M|}{T_{\text {effective }}} .
$$

(The outgoing radiation, since it is not exactly Planckian, should really be analyzed using non-equilibrium thermodynamics; but use of the effective temperature is a well-known stand-in for such effects.) Overall one has

$$
\mathrm{d} S_{\text {total }}=|\mathrm{d} M|\left(\frac{1}{T_{\text {effective }}}-\frac{1}{T_{H}}\right) \geq 0 .
$$


So the Hawking evaporation process actually increases the total entropy of the universe. (Note this is intrinsically a coarse-graining entropy associated with throwing away detailed information regarding the Hawking flux; this argument has nothing to say one way or another regarding the unitarity of the underlying physical process.) This is perhaps somewhat unexpected from the standard point of view, but is utterly unavoidable as soon as one takes proper cognisance of greybody, adiabaticity, and phase space effects.

\section{Wick rotation and the Hawking flux}

Ultimately the origin of the often-made but mistaken assertion that the Hawking flux is exactly Planckian seems to trace back to an over-enthusiastic and uncritical adoption of Wick rotation (Euclidean quantum gravity) techniques [37]. Certainly the Wick rotation of a static black hole $\left(t \rightarrow i t\right.$, in the manifestly static coordinate system where $\left.g_{t i}=0\right)$, combined with the condition that there be no conical singularity at the Euclideanized version of the Lorentzian-signature Killing horizon, picks out the surface gravity as being physically important, being related (via periodicity in imaginary time) to a notion of temperature but this is by construction an intrinsically equilibrium argument for a black hole in exact thermal equilibrium with a heat bath at the Hawking temperature [37].

- By construction the heat bath has an exactly Planckian spectrum, simply because it is assumed to be in exact thermal equilibrium; the greybody factors quietly drop out.

- By construction the situation is static; there simply are no adiabaticity conditions since $\dot{\kappa} \equiv 0$ exactly.

- By construction there are no phase-space constraints; since (typically) one is completely ignoring back-reaction.

But this Wick-rotated Euclideanized system tells you relatively little regarding the nonequilibrium emission of the Hawking flux into vacuum; the Unruh quantum vacuum state is radically different from the Hartle-Hawking quantum vacuum state. (Wick rotation automatically puts one into the Hartle-Hawking quantum vacuum state, not the physically relevant Unruh vacuum state.) While the Wick rotation trick provides a "quick and dirty" way of relating surface gravity to Hawking temperature [38], it misses much of the essential physics of the evaporation process. Once one considers a real black hole evaporating into vacuum, the Hawking flux is no longer exactly Planckian - the shape of the spectrum must at the very least be modified by the three physical effects considered above.

\section{Correlations in the Hawking flux}

Are the Hawking quanta in any way correlated with each other? This quite deceptively innocent question can easily initiate a firestorm of quite inconclusive debate.

The original 1850's notions of thermality, based as they were on entirely classical thermodynamic reasoning applied to black bodies, (such as, for instance, the traditional "leaky cavity" or "lamp-black" surfaces), made no intrinsic assumptions regarding the possibility 
of correlations in the outgoing radiation. But modern abuse of the word "thermal" often implicitly makes assumptions about a lack of correlations. It is essential to realise that the physical distinction between "Planckian" (Planck-shaped spectrum) and "thermal" is both important and subtle. No-one seriously doubts that burning a lump of coal in a leaky furnace results in an approximately Planckian spectrum, (an approximately "black body" spectrum), nor that this process implies correlations in the outgoing radiation — which then cannot be exactly "thermal" in the technical sense that this word has come to be used (or rather abused) in the modern literature.

Now Hawking's original 1974 calculation [1, 2], (and its modern adiabatic variants, see for instance references [26] and [27]), certainly demonstrate that a collapsing ball of matter will excite the quantum vacuum state, and that the outgoing radiation is approximately blackbody, that is, has an approximately Planck-shaped spectrum, (at least up to greybody, adiabaticity, and phase space effects, as discussed above). But the considerably stronger statement that the outgoing Hawking quanta are completely uncorrelated, (the modern misuse of the word "thermal"), depends on a separate and very much stronger implicit assumption: that in a semi-classical astrophysical black hole an event horizon forms to permanently hide any possible correlations, in such a way that they never again become visible to the external universe - but the possibility of doing this depends on delicate issues of global geometry - including what will happen in the infinite future [25]. In contrast apparent horizons or trapping horizons, while they may temporarily hide correlations, do not necessarily do so permanently. Without an event horizon, whose very existence is delicately predicated on assumptions being made about the infinite future, a black hole defined in terms of apparent or trapping horizons will behave much more like a furnace; a leaky furnace with a small hole in it, the original 1850's classical thermodynamic definition of a "black body".

\section{Analogue Hawking flux}

To really drive home the point that the existence of possible correlations in the Hawking flux is logically independent from the existence of the Hawking flux itself simply consider an acoustic black hole (dumb hole) [39]. (For various theoretical developments see [40-46] and [47-49]. For a laboratory implementation using surface waves see $[4,5]$. For a more recent laboratory implementation using BECs see [6].)

There is widespread agreement that an acoustic horizon (defined by the normal component of fluid velocity exceeding the local speed of sound) will emit an approximately Planckian spectrum of Hawking phonons; but there is absolutely no requirement that the acoustic horizon be an event horizon - in fact by accelerating or decelerating the fluid flow it is easy to make acoustic horizons appear and disappear at will. Any horizon that can completely disappear (without any trace of its prior existence, and without any way of permanently hiding correlations) will qualitatively behave like a leaky furnace with a small hole in it, the original 1850's classical thermodynamic definition of a "black body". So in these analogue systems, not only is there no reason to believe that there is any "information puzzle", but in contrast there is every reason to believe that ordinary unitary evolution and standard physics applies. 
Consequently, even if one could somehow prove that the Hawking photons coming from a specifically general relativistic black hole were uncorrelated, this would merely be a sideeffect of the specific details of general relativistic black holes, (as opposed to the generic features of analogue black holes); it would have nothing to do with the fundamental physics underlying Hawking radiation itself. In short:

- Hawking radiation is associated with the apparent/trapping horizon, and couldn't care less about the event horizon (if present).

- Unitarity violation (if present) is associated with the event horizon (if present), and couldn't care less about the apparent/trapping horizon.

\section{Discussion}

In short, the so-called "information puzzle", (often somewhat excessively referred to as the "information paradox"), is intimately reliant on the assumed existence of an event horizon, and much of the force of the information puzzle simply goes away once one uses apparent horizons or trapping horizons to define what we mean by a black hole [25, 50, 51]. This observation is closely related to Hawking's recent arguments regarding the necessity of making careful physical distinctions between the mathematical concepts of event horizon and apparent horizon [50]: "The absence of event horizons means that there are no black holes - in the sense of regimes from which light can't escape to infinity. There are, however, apparent horizons which persist for a period of time." Similarly, a decade ago Hawking asserted [51]: "The way the information gets out seems to be that a true event horizon never forms, just an apparent horizon."

The physical picture that then emerges matches quite nicely with certain proposals for the Hawking radiation process, both somewhat older and more recent, that make no intrinsic reference to event horizons per se [52-61]. Exact thermality of the Hawking flux, and a total absence lack of correlations in the Hawking flux, is often asserted in the scientific literature - but neither assertion holds up to any level of scrutiny. The spectrum of the Hawking flux is certainly not exactly Planckian, and the effective temperature (suitably defined) of the Hawking flux is not equal to, but is instead bounded above, by the Hawking temperature. Whether or not correlations exist in the Hawking flux is contingent upon the assumed existence of event horizons (as opposed to apparent/ trapping horizons). Certainly event horizons are not necessary for the development of a Hawking flux, and the often assumed survival of classical event horizons in semi-classical physics is an assumption that is increasingly in doubt $[25,50,51]$.

There is a crucial difference between the "qualitative" and "quantitative" information loss problems.

- The "qualitative" problem is this: if a spacelike singularity forms (in the strict mathematical sense), then there will be a (strict mathematical) event horizon, and unavoidably some loss of unitarity associated with any matter that might cross the event horizon. 
- The "quantitative" problem is this: How much information is lost behind the event horizon, (if it forms), and how much comes out in the Hawking radiation? ${ }^{4}$

Only if you assume that the event horizon actually forms, and that it closely tracks the apparent/trapping horizon, is there ever any significant information loss.

\section{Acknowledgments}

This research was supported by the Marsden Fund, and by a James Cook fellowship, both administered by the Royal Society of New Zealand.

Open Access. This article is distributed under the terms of the Creative Commons Attribution License (CC-BY 4.0), which permits any use, distribution and reproduction in any medium, provided the original author(s) and source are credited.

\section{References}

[1] S.W. Hawking, Black hole explosions, Nature 248 (1974) 30 [InSPIRE].

[2] S.W. Hawking, Particle creation by black holes, Commun. Math. Phys. 43 (1975) 199 [Erratum ibid. 46 (1976) 206] [INSPIRE].

[3] S.W. Hawking, Breakdown of predictability in gravitational collapse, Phys. Rev. D 14 (1976) 2460 [INSPIRE].

[4] S. Weinfurtner, E.W. Tedford, M.C.J. Penrice, W.G. Unruh and G.A. Lawrence, Measurement of stimulated Hawking emission in an analogue system, Phys. Rev. Lett. 106 (2011) 021302 [arXiv: 1008.1911] [INSPIRE].

[5] S. Weinfurtner, E.W. Tedford, M.C.J. Penrice, W.G. Unruh and G.A. Lawrence, Classical aspects of Hawking radiation verified in analogue gravity experiment, Lect. Notes Phys. $\mathbf{8 7 0}$ (2013) 167 [INSPIRE].

[6] J. Steinhauer, Observation of self-amplifying Hawking radiation in an analog black hole laser, Nature Phys. 10 (2014) 864 [arXiv:1409.6550] [InSPIRE].

[7] A. Almheiri, D. Marolf, J. Polchinski and J. Sully, Black holes: complementarity or firewalls?, JHEP 02 (2013) 062 [arXiv:1207.3123] [INSPIRE].

[8] A. Almheiri, D. Marolf, J. Polchinski, D. Stanford and J. Sully, An apologia for firewalls, JHEP 09 (2013) 018 [arXiv: 1304.6483] [INSPIRE].

[9] S.L. Braunstein, Black hole entropy as entropy of entanglement, or it's curtains for the equivalence principle, arXiv:0907.1190v1.

[10] S.L. Braunstein, S. Pirandola and K. Życzkowski, Better late than never: information retrieval from black holes, Phys. Rev. Lett. 110 (2013) 101301.

[11] R. Bousso, Complementarity is not enough, Phys. Rev. D 87 (2013) 124023 [arXiv:1207.5192] [INSPIRE].

[12] Y. Nomura, J. Varela and S.J. Weinberg, Complementarity endures: no firewall for an infalling observer, JHEP 03 (2013) 059 [arXiv: 1207.6626] [INSPIRE].

\footnotetext{
${ }^{4}$ Very recent articles specifically addressing this specific point include those by Brustein and Medved [62, 63], and by Saini and Stojkovic [64]. In these articles it is the quantitative estimates of the information budget that are important.
} 
[13] S.D. Mathur and D. Turton, Comments on black holes I: the possibility of complementarity, JHEP 01 (2014) 034 [arXiv: 1208.2005] [INSPIRE].

[14] T. Banks and W. Fischler, Holographic space-time does not predict firewalls, arXiv: 1208.4757 [INSPIRE].

[15] R. Brustein, Origin of the blackhole information paradox, Fortsch. Phys. 62 (2014) 255 [arXiv: 1209.2686] [INSPIRE].

[16] S. Hossenfelder, Comment on the black hole firewall, arXiv:1210.5317 [INSPIRE].

[17] K. Larjo, D.A. Lowe and L. Thorlacius, Black holes without firewalls, Phys. Rev. D 87 (2013) 104018 [arXiv: 1211.4620] [INSPIRE].

[18] S.B. Giddings, Nonviolent nonlocality, Phys. Rev. D 88 (2013) 064023 [arXiv:1211.7070] [INSPIRE].

[19] E. Verlinde and H. Verlinde, Passing through the firewall, arXiv:1306.0515 [InSPIRE].

[20] D.N. Page, Is black hole evaporation predictable?, Phys. Rev. Lett. 44 (1980) 301 [INSPIRE].

[21] S.W. Hawking, The unpredictability of quantum gravity, Commun. Math. Phys. 87 (1982) 395 [INSPIRE].

[22] T. Banks, L. Susskind and M.E. Peskin, Difficulties for the evolution of pure states into mixed states, Nucl. Phys. B 244 (1984) 125 [inSPIRE].

[23] J. Preskill, Do black holes destroy information?, hep-th/9209058 [INSPIRE].

[24] S.W. Hawking, Virtual black holes, Phys. Rev. D 53 (1996) 3099 [hep-th/9510029] [INSPIRE].

[25] M. Visser, Physical observability of horizons, Phys. Rev. D 90 (2014) 127502 [arXiv:1407.7295] [INSPIRE].

[26] C. Barceló, S. Liberati, S. Sonego and M. Visser, Minimal conditions for the existence of a Hawking-like flux, Phys. Rev. D 83 (2011) 041501 [arXiv:1011.5593] [InSPIRE].

[27] C. Barceló, S. Liberati, S. Sonego and M. Visser, Hawking-like radiation from evolving black holes and compact horizonless objects, JHEP 02 (2011) 003 [arXiv: 1011.5911] [INSPIRE].

[28] D.N. Page, Particle emission rates from a black hole. 1. Massless particles from an uncharged, nonrotating hole, Phys. Rev. D 13 (1976) 198 [INSPIRE].

[29] D.N. Page, Particle emission rates from a black hole. 2. Massless particles from a rotating hole, Phys. Rev. D 14 (1976) 3260 [INSPIRE].

[30] J.D. Bekenstein and A. Meisels, Einstein A and B coefficients for a black hole, Phys. Rev. D 15 (1977) 2775 [INSPIRE].

[31] J. Escobedo, Greybody factors: hawking radiation in disguise, M.Sc. Thesis, University of Amsterdam, Amsterdam, The Netherlands (2008).

[32] P. Boonserm and M. Visser, Bounding the greybody factors for Schwarzschild black holes, Phys. Rev. D 78 (2008) 101502 [arXiv:0806.2209] [inSPIRE].

[33] P. Boonserm, T. Ngampitipan and M. Visser, Regge-Wheeler equation, linear stability and greybody factors for dirty black holes, Phys. Rev. D 88 (2013) 041502 [arXiv: 1305.1416] [INSPIRE].

[34] M. Visser, Hawking radiation: a particle physics perspective, Mod. Phys. Lett. A 8 (1993) 1661 [hep-th/9204062] [INSPIRE].

[35] M.K. Parikh and F. Wilczek, Hawking radiation as tunneling, Phys. Rev. Lett. 85 (2000) 5042 [hep-th/9907001] [INSPIRE]. 
[36] J.D. Bekenstein, Black holes and entropy, Phys. Rev. D 7 (1973) 2333 [InSPIRE].

[37] G.W. Gibbons and S.W. Hawking, Action integrals and partition functions in quantum gravity, Phys. Rev. D 15 (1977) 2752 [InSPIRE].

[38] M. Visser, Dirty black holes: thermodynamics and horizon structure, Phys. Rev. D 46 (1992) 2445 [hep-th/9203057] [INSPIRE].

[39] W.G. Unruh, Experimental black hole evaporation, Phys. Rev. Lett. 46 (1981) 1351 [INSPIRE].

[40] M. Visser, Acoustic propagation in fluids: an unexpected example of Lorentzian geometry, gr-qc/9311028 [INSPIRE].

[41] M. Visser, Acoustic black holes: horizons, ergospheres and Hawking radiation, Class. Quant. Grav. 15 (1998) 1767 [gr-qc/9712010] [INSPIRE].

[42] C. Barcelo, S. Liberati and M. Visser, Analogue gravity, Living Rev. Rel. 8 (2005) 12 [Living Rev. Rel. 14 (2011) 3] [gr-qc/0505065] [InSPIRE].

[43] M. Visser, Survey of analogue spacetimes, Lect. Notes Phys. 870 (2013) 31 [arXiv: 1206.2397] [INSPIRE].

[44] C. Barcelo, S. Liberati, S. Sonego and M. Visser, Causal structure of acoustic spacetimes, New J. Phys. 6 (2004) 186 [gr-qc/0408022] [inSPIRE].

[45] C. Barcelo, S. Liberati, S. Sonego and M. Visser, Hawking-like radiation does not require a trapped region, Phys. Rev. Lett. 97 (2006) 171301 [gr-qc/0607008] [INSPIRE].

[46] M. Visser, Hawking radiation without black hole entropy, Phys. Rev. Lett. 80 (1998) 3436 [gr-qc/9712016] [INSPIRE].

[47] C. Barcelo, S. Liberati and M. Visser, Probing semiclassical analog gravity in Bose-Einstein condensates with widely tunable interactions, Phys. Rev. A 68 (2003) 053613 [cond-mat/0307491] [INSPIRE].

[48] C. Barcelo, S. Liberati and M. Visser, Towards the observation of Hawking radiation in Bose-Einstein condensates, Int. J. Mod. Phys. A 18 (2003) 3735 [gr-qc/0110036] [INSPIRE].

[49] C. Barcelo, S. Liberati and M. Visser, Analog gravity from Bose-Einstein condensates, Class. Quant. Grav. 18 (2001) 1137 [gr-qc/0011026] [INSPIRE].

[50] S.W. Hawking, Information preservation and weather forecasting for black holes, arXiv: 1401.5761 [INSPIRE].

[51] S.W. Hawking, Black holes and the information paradox, abstract of the talk given at the $17^{\text {th }}$ International Conference on General Relativity And Gravitation (GR17), July 18-24, Dublin, Ireland (2004).

[52] T.A. Roman and P.G. Bergmann, Stellar collapse without singularities?, Phys. Rev. D 28 (1983) 1265 [inSPIRE].

[53] M.K. Parikh and F. Wilczek, Global structure of evaporating black holes, Phys. Lett. B 449 (1999) 24 [gr-qc/9807031] [INSPIRE].

[54] A. Ashtekar and M. Bojowald, Black hole evaporation: a paradigm, Class. Quant. Grav. 22 (2005) 3349 [gr-qc/0504029] [INSPIRE].

[55] S.A. Hayward, Formation and evaporation of regular black holes, Phys. Rev. Lett. 96 (2006) 031103 [gr-qc/0506126] [INSPIRE].

[56] S.A. Hayward, The Disinformation problem for black holes (conference version), gr-qc/0504037 [INSPIRE]. 
[57] M. Visser, Black holes in general relativity, PoS (BHGRS) 001 [arXiv:0901.4365] [INSPIRE].

[58] S. Hossenfelder and L. Smolin, Conservative solutions to the black hole information problem, Phys. Rev. D 81 (2010) 064009 [arXiv:0901.3156] [INSPIRE].

[59] V.P. Frolov, Information loss problem and a 'black hole' model with a closed apparent horizon, JHEP 05 (2014) 049 [arXiv: 1402.5446] [INSPIRE].

[60] W. Israel, A Massless Firewall, arXiv:1403.7470 [INSPIRE].

[61] J.M. Bardeen, Black hole evaporation without an event horizon, arXiv:1406.4098 [INSPIRE].

[62] R. Brustein and A.J.M. Medved, How black holes burn: Entanglement entropy evolution for an evaporating black hole, Phys. Rev. D 91 (2015) 084062 [arXiv:1407.4914] [InSPIRE].

[63] R. Brustein and A.J.M. Medved, Black hole firewalls, smoke and mirrors, Phys. Rev. D 90 (2014) 024040 [arXiv:1401.1401] [INSPIRE].

[64] A. Saini and D. Stojkovic, Radiation from a collapsing object is manifestly unitary, Phys. Rev. Lett. 114 (2015) 111301 [arXiv:1503.01487] [INSPIRE]. 